\title{
Changes in intracranial CSF volume after lumbar puncture and their relationship to post-LP headache
}

\author{
R Grant, B Condon, I Hart, G M Teasdale
}

\begin{abstract}
Post-lumbar puncture (LP) headache may be due to "low CSF pressure", leading to stretching of pain sensitive intracranial structures. The low intracranial pressure is secondary to net loss of intracranial CSF. It has, however, not been possible to measure intracranial CSF volume accurately during life until recently. Intracranial CSF volume can now be measured non-invasively by a MRI technique. The changes in intracranial CSF volume were studied in 20 patients who had LP. Total intracranial CSF volume was reduced in 19 of the 20 patients 24 hours after LP (range -1.8 mls to $-158.6 \mathrm{mls})$. Most of the CSF was lost from the cortical sulci. Very large reductions in intracranial CSF volume were frequently related to post-LP headache but some patients developed headache with relatively little alteration in the intracranial CSF volume. There was not a measurable change in position of the intracranial structures following LP.
\end{abstract}

The pathophysiological basis of the headache that commonly follows lumbar puncture remains uncertain. Wolff suggests that the pain sensitive structures are stretched due to "sagging" of the brain. ${ }^{1}$ An alternative theory is that the volume of the intracranial CSF lost following lumbar puncture is to some extent compensated by an increase in the intracranial blood volume which in turn causes headache. These theories have not been tested until recently because it has not been possible to measure intracranial CSF volume during life. ${ }^{2}$

Using an MRI technique, ${ }^{2}$ we measured the volume of intracranial CSF in 20 patients before and 24 hours after lumber puncture. Our aim was to discover if there is a relationship between the change in volume of intracranial CSF and the occurrence of headache after lumbar puncture. Midline sagittal cranial MRI scans were also performed before and after LP to define the anatomical relationship between the posterior fossa structures and the cranio-cervical junction, and hence to determine if significant displacement occurred after LP.

\section{Subjects and methods}

Twenty patients with neurological symptoms who were admitted for elective investigation, including lumbar puncture, were studied. There were eight males aged from $25-71$ years (mean 41.5 years) and 12 females aged from 14-64 years (mean 38.5 years). Each patient underwent measurements of their intracranial CSF volume, before LP and 24 hours afterwards. Each patient gave written informed consent for MRI. Patients were excluded if they were being investigated for headaches, if they complained of headache at the time of admission or if they were taking analgesics. Patients were excluded if they were having contrast mylography. At the time of the second MRI scan, the patient was questioned about headache. Patients were contacted also three to four weeks later to determine whether they had experienced headache and, if so, its severity and duration.

Spinal puncture was performed with the patient in the curled lateral recumbent position. All punctures were performed in the lumbar region with an 18 gauge needle. The CSF "opening" pressure was measured using a clinical manometer, $10 \mathrm{mls}$ of CSF were collected for analysis and CSF "closing" pressure was then measured. The CSF was sent for biochemical, neuroimmunological and bacteriological analysis. The patient was advised to remain in bed for at least $\mathbf{1 4}$ hours.

\section{Intracranial CSF volume measurement}

A special pulse sequence (IRCP 300/400/ 5000) was used which provides an image of CSF only. ${ }^{2}$ Two images were obtained in the sagittal plane. The first had a slice thickness of $240 \mathrm{~mm}$ and therefore included signal from all the CSF in the head. The slice thickness of the second was varied to include the lateral ventricles but to exclude the overlying and underlying cortical sulci. The signal intensity from a desired region of interest was directly proportional to the volume of CSF in that region (area $\mathrm{x}$ slice thickness) and the signal intensity was compared with that from a reference phial containing a known volume of normal saline.

Total intracranial CSF volume ( $\mathrm{tCSF}(\mathrm{v}))$, ventricular CSF volume (vCSF(v)), cortical sulcal CSF volume $(\operatorname{csCSF}(v))$ and posterior fossa CSF volume ( $\mathrm{pfCSF}(\mathrm{v}))$ measurements were performed as previously described by Condon. ${ }^{2}$

The anatomical relationship between the cerebellar tonsils and the foramen magnum was imaged before and 24 hours after LP in five subjects using an $8 \mathrm{~mm}$ thick multislice sagittal MRI sequence (SE40/500). Any 
change in the position of the cerebellar tonsils with respect to the foramen magnum was recorded.

\section{Results}

Eleven of the 20 patients complained of headache when questioned at the time of the second scan. At follow up, two patients complained of very severe protracted headache lasting at least three weeks.

Before LP the median $\operatorname{tCSF}(v)$ was significantly greater in males $(224.8 \mathrm{mls})$ than in females $(137.8 \mathrm{mls})$ and was correlated with age in both sexes (males $r=0.77$, females $r=$ 0.74). Twenty four hours after $\operatorname{LP}, \operatorname{tCSF}(v)$ had decreased in 19 of the 20 patients (range -1.8 to $-158.6 \mathrm{mls}$ ) (Figure). The $\operatorname{csCSF}(\mathrm{v})$ also decreased in 19 of the 20 patients, the $v C S F(v)$ in 16 and the $\operatorname{pfCSF}(v)$ in 13 patients. One patient had an increase of $8.8 \mathrm{mls}$ in $\operatorname{tCSF}(v)(+1.6 \mathrm{mls}$ in the $\operatorname{csCSF}(v),+5.3 \mathrm{mls}$ in the $\operatorname{vCSF}(v)$ and $+1.9 \mathrm{mls}$ in the $\operatorname{pfCSF}(\mathrm{v}))$. This patient and two other patients had been advised by their doctor to lie prone for 24 hours after LP. The tCSF $(v), \operatorname{csCSF}(v)$ and the $\mathrm{vCSF}(\mathrm{v})$ all reduced significantly after the LP (Wilcoxon signed ranks test $p<0.01$ ); however, the pf CSF(v) did not alter significantly. The medians and interquartile ranges for the differences are given in the table. By far the largest proportion of CSF was lost from the cortical sulci. From examining the scans this seemed to be predominantly due to loss of CSF from the cortical sulci laterally, from over the cerebral hemispheres and from the Sylvian fissure rather than from the interhemispheric fissure. The change in $\operatorname{tCSF}(v)$ did not correlate with the age or sex of the patient, or with the initial $\operatorname{tCSF}(v)$ ( $\mathrm{tCSF}(\mathrm{v})$ :males $\mathbf{r}=$ $0 \cdot 218$, females $r=0 \cdot 201$ ).

There was no measurable change in position of the cerebellar tonsils in the patients who had sagittal SE40/500 imaging before and 24 hours after LP.

Figure Changes in total intracranial CSF volume following $L P$.

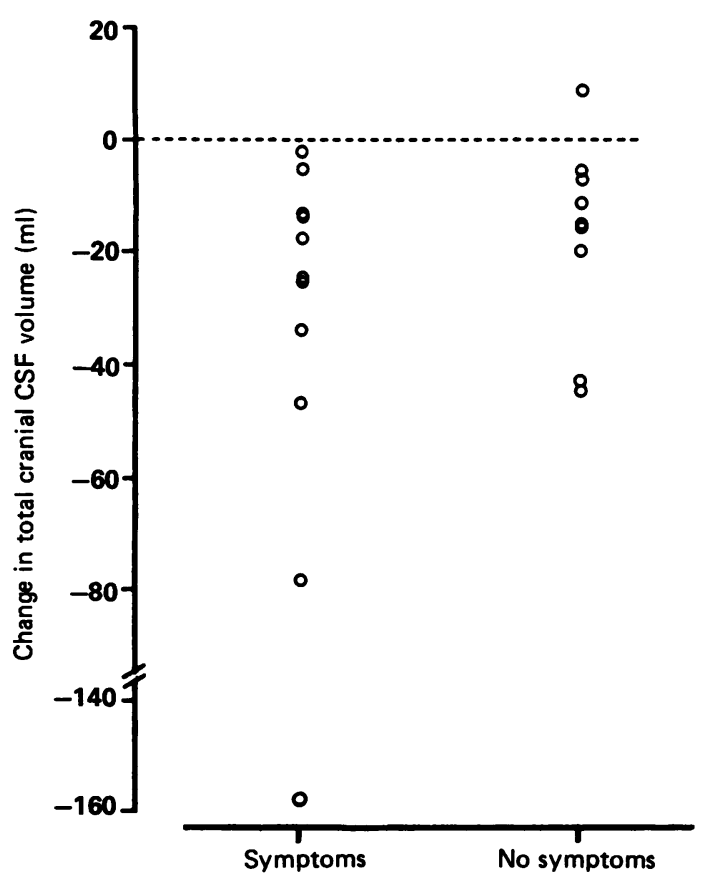

Table Group changes in intracranial CSF volume following $L P$

\begin{tabular}{llll}
\hline & $\begin{array}{l}\text { Lower } \\
\text { Quartile }\end{array}$ & Median & $\begin{array}{l}\text { Upper } \\
\text { Quartile }\end{array}$ \\
\hline Change in $\operatorname{CCSF}(\mathrm{v})$ & $-33 \cdot 7$ & -16.5 & $-11 \cdot 1$ \\
Change in $\operatorname{csCSF}(\mathrm{v})$ & $-30 \cdot 6$ & -15.6 & -7.9 \\
Change in vCSF(v) & $-2 \cdot 2$ & -0.95 & -0.3 \\
Change in pfCSF(v) & $-4 \cdot 1$ & -1.2 & -1.9 \\
\hline
\end{tabular}

Headache developed in six of eight patients whose tCSF(v) decreased by more than $20 \mathrm{mls}$ compared with five of 12 patients who lost less than $20 \mathrm{mls}$ of CSF following LP. The difference, however, between the volume change in patients with and without headache did not reach statistical significance (Wilcoxon signed ranks test $p>0.05$ ). Two patients who had SE 40/500 midline sagittal scans before and after LP developed headache and three did not. There was no obvious change in position of the intracranial structures in any set of scans.

\section{Discussion}

This study demonstrates the wide range of the volume of intracranial CSF and the very variable alterations in intracranial CSF volume that can occur as a result of lumbar puncture. Total intracranial CSF volume is almost always reduced following LP and the majority of the deficit is due to loss of cortical sulcal CSF. This may explain why subdural haematomas can occur as a complication of LP. The changes in total intracranial CSF volume may indeed be an underestimate of the difference in the standing position, since the scans were performed with the patient recumbent, when CSF may be displaced into the cranium from the spinal subarachnoid space.

The incidence of headache in this study $(55 \%)$ is higher than in other studies ${ }^{34}$ and is probably related to the larger gauge of the LP needle used. ${ }^{5}$ The mechanisms that cause postLP headache are still debatable. Rapid removal of around $20 \mathrm{mls}$ of CSF (approximately 15\% of the estimated CSF volume in healthy volunteers) results in headache. As the headache becomes evident on standing and usually settles on lying down, it has been postulated that a reduction in intracranial pressure when erect produces "sagging" of the brain and traction on pain sensitive structures with resultant headache. However, in studies where the CSF pressure was measured following lumbar puncture, there was no clear relationship between the degree of reduction in CSF pressure and the presence of headache. Moreover, from the MRI performed in this study there was not any evidence of "sagging" of the brain. An alternative theory is that the post-LP headache is related to vasodilatation. There is a reciprocal relationship between intracranial CSF volume and intracranial blood volume (assuming no change in brain volume)-the modified Monro-Kellie doctrine. ${ }^{7}$ This is supported by the reciprocal CSF volume changes in response to cerebral vasodilatation induced by hypercapnia measured by the MRI method. ${ }^{8}$ Avezatt and Eijndhoven have shown that the CSF compartment can cope with a volume load by 
"volume storage" and "volume compensation". 9 Volume storage occurs as a result of changes in compliance of the arachnoid and dural tissues "buffering" the change in CSF volume. Volume compensation occurs where the change in CSF volume is balanced by a reciprocal change in one of the other two volume compartments in the cranium (blood volume or brain volume). It therefore seems likely that the intracranial CSF volume can also cope with a CSF deficit by similar mechanisms, namely, compliance of the system with a resulting reduction in the volume pressure relationship and volume compensation due to an increase in intracranial blood volume. Most of the volume compensation must be accommodated by the cerebral venous drainage pathways rather than by arterial vasodilatation. The headache that follows LP closely resembles that induced by inhalation of $\mathrm{CO}^{6}$ and is also worsened by jugular compression, ${ }^{11}$ the inhalation of ergotamine tartrate ${ }^{10}$ and compression of the carotid artery in the neck. These features and the "throbbing" character of the headache may be more in keeping with arterial vasodilatation.

This study demonstrated a trend towards a relationship between intracranial CSF volume and post-LP headache. In the numbers studied this failed to reach statistical significance. Nevertheless, it is likely that changes in CSF volume play an important role in the pathogenesis of post-LP headache. Measurements of cerebral blood volume before and after LP may be helpful in either refuting or corroborating this view.

1 Wolff HG. Headache and other head pain, 2nd ed. New York: Oxford University Press 1963, 96-125.

2 Condon B, Patterson J, Wyper D, et al. Intracranial CSF volumes determined using Magnetic Resonance Imaging. Lancet 1986;i:1355-8.

3 Marton KI, Gean AD. The spinal tap: a new look at an old test. Ann Intern Med 1986;104:840-8.

4 Vandam LD, Dripps RD. Long term follow up of patients who received 10,098 spinal anaesthetics. Syndrome of who received 10,098 spinal anaesthetics. Syndrome of

5 Toutellotte WW, Haerer AF, Heller GL, et al. Post lumbar puncture headaches. Springfield III: Charles C Thomas 1963, 79-97.

6 Alksne JF. Headaches associated with changes in intracranial pressure. In: Dalessio DJ, ed. Wolff's Headache and head pain, 4th ed. New York: Oxford University Press 1980, 301-13.

7 Burrows G. Disorders of the cerebral circulation, London 1846. From: Lundberg N. The saga of the Monro-Kellie doctrine. In: Ishii S, Nagi $H$, Brock $M$, eds. Intracranial pressure V. Berlin: Springer Verlag, 1983, 68-75.

$8 \mathrm{Grant} \mathrm{R}$, Condon B, Patterson J, et al. Physiological changes in the cranial CSF volume: The effects of hypercapnia and hypocapnia. J Neurol Neurosurg Psychiatry 1989;52: 218-22.

9 Avezaat CJ, Eijndhoven JHM van. How does the intracranial system cope with a disturbance of its volume? In: Miller JD, Teasdale GM, Rowan JD, Galbraith SL, Mendelow AD, eds. Intracranial pressure VI. Berlin: Springer Verlag, 1986, 99-104.

10 Guttman SA (Personal communication to Wolff). In: Dalessio DJ, ed. Wolffs Headache and head pain, 2nd ed. New York: Oxford University Press 1966, 96-125.

11 Pickering GW. Lumbar puncture headache. Brain 1948 71:274-80. 